\title{
ERFOLGSSTORY KRÄUTERMEDIZIN
}

\section{Sehr geehrte Frau Kollegin! \\ Sehr geehrter Herr Kollege!}

Die TCM-Kräutermedizin hat in den letzten Jahren eine unglaubliche Erfolgsstory erlebt. Im Jahrgang 2004-2005 wurden die ersten Lehrgänge, die von der Österreichischen Ärztekammer anerkannt wurden, absolviert. In der Zwischenzeit haben österreichweit an die 500 KollegInnen das entsprechende ÖÄK-Diplom erworben, womit eine Versorgung der interessierten PatientInnen weitgehend gewährleistet ist. Parallel dazu hat sich in der öffentlichen Meinung und auch in der Einschätzung der schulmedizinischen KollegInnen die TCM als sinnvolle Therapiemöglichkeit absolut etablieren können. Man stößt in der Recherche über TCM-Diagnostik und -Therapie fast ausschließlich auf positive Kritiken. Diese erfreuliche Entwicklung fand ihren Höhepunkt in der Verleihung des letztjährigen Nobelpreises für die Entdeckung der wertvollen Wirkung von Artimisin in der Malariatherapie einem wertvollen Beitrag derTCM für die Weltgesundheit.

Ich lade Sie, sehr geehrte Frau Kollegin und sehr geehrter Herr Kollege, ganz herzlich ein, diese Methodik für sich persönlich zu entdecken und in diesem Herbst in unsere Ausbildung zur TCM-Diagnostik und -Arzneimitteltherapie einzusteigen. Ein neues Universum an therapeutischen Möglichkeit zum Nutzen Ihrer PatientInnen wird sich darin für Sie erschließen.

Ich wünsche Ihnen allen einen schönen Herbst und eine anregende Lektüre dieser Zeitschrift

Ihr

Prof. Dr. med. Leopold Dorfer

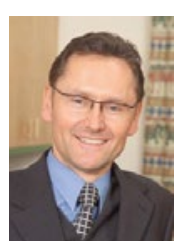

Prof. Dr. med. Leopold Dorfer

Präsident der Österreichischen Gesellschaft für Kontrollierte Akupunktur und TCM (OGKA)

Glacisstraße 7, A-8010 Graz

Tel. +43 316/374050

E-Mail: office@ogka.at, Internet:www.ogka.at 